\title{
Towards the entropy of gravity time-dependent models via the Cardy-Verlinde formula
}

\author{
Octavio Obregón* \\ Department of Applied Mathematics and Theoretical Physics \\ Wilberforce Road, Cambridge CB3 OWA, U.K. \\ Leonardo Patiño and Hernando Quevedd \\ Instituto de Ciencias Nucleares, Universidad Nacional Autónoma de México \\ P.O. Box 70-543, 04510 México D.F., México
}

(Dated: November 17, 2018)

\begin{abstract}
For models with several time-dependent components generalized entropies can be defined. This is shown for the Bianchi type IX model. We first derive the Cardy-Verlinde formula under the assumption that the first law of thermodynamics is valid. This leads to an explicit expression of the total entropy associated with this type of universes. Assuming the validity of the Cardy entropy formula, we obtain expressions for the corresponding Bekenstein, Bekenstein-Hawking and Hubble entropies. We discuss the validity of the Cardy-Verlinde formula and possible extensions of the outlined procedure to other time-dependent models.
\end{abstract}

PACS numbers: 11.25.Hf, 98.80.Jk, 04.20.Jb

\footnotetext{
*Permanent address: Instituto de Física de la Universidad de Guanajuato, P.O. Box E-143, 37150 León Gto., México; Electronic address: octavio@ifug3.ugto.mx

${ }^{\dagger}$ Electronic address: jaidar, quevedo@nuclecu.unam.mx
} 


\section{INTRODUCTION}

The concept of entropy for cosmological models has many subtleties. One usually starts by demanding that the thermodynamic system described by the corresponding time-dependent model satisfies the first law of thermodynamics. Then, one considers a specific equation of state and imposes compatibility between field equations and the first law of thermodynamics. As a result one usually obtains explicit expressions for the thermodynamic variables (temperature and entropy) of the model. In a further step, the question is addressed about the physical relevance of the state variables, the equation of state linking them, and the thermodynamic variables. In the case of a simple Friedman-Robertson-Walker (FRW) universe this procedure can successfully be performed [1], yielding physically reasonable thermodynamic and state variables.

Recently, a new approach has been proposed by Verlinde [2] who noticed a formal relationship between the field equations for a FRW cosmology and the thermodynamic formulas of conformal field theory (CFT). Although at first sight this seems to be only a remarkable coincidence, a deeper analysis seems to link these results with the novel conceptual ideas inherent in the holographic principle.

The conceptual origin of the holographic principle was settled in the 70's by Bekenstein 3] and Hawking [4], who formulated the second law of thermodynamics for black holes and the process of particle creation by black holes, respectively. These ideas were implemented in the context of quantum field theories, specially in quantum gravity and cosmology by t'Hooft [5] and Susskind and Fischler [6, 7]. The holographic principle is a statement about the counting of the quantum states of a physical system. It states that the degrees of freedom contained in a given spatial volume can be encoded on its boundary and that the density on the boundary does not exceed one degree of freedom per Planck area. This is inspired by the behavior of black hole entropy, that the number of degrees of freedom of a gravitating system in a region $L$ of space is the same as that of a system on the boundary of $L$. This assumption has as a consequence also that the number of degrees of freedom should grow only slower than volume. These restrictions are essentially non local and would require ultimately profound reformulations of our description of fields and their interactions.

The main ideas of the holographic principle have been used recently in the context of gravity, string theory, and CFT's. In particular, the Cardy formula [8], which allows the 
counting of quantum states in a two-dimensional conformal field theory, has been generalized by Verlinde [2] to include arbitrary spacetime dimensions and determine the density of states. This result is now commonly referred to as the Cardy-Verlinde formula. Verlinde also proposed that a closed universe has a Casimir contribution to its energy and entropy and that the Casimir energy is bounded from above by the Bekenstein-Hawking energy. Only for the case of a radiation dominated universe this bound leads to a unification of the Bekenstein and Hubble entropy bounds for weakly and strongly self-gravitating universes, respectively. Furthermore, the Cardy-Verlinde formula turns out to coincide with the Friedman equation at the moment when the bound on the Casimir energy becomes saturated. These results give rise to the natural question: Is this formal merging between the Friedman formula, the Cardy entropy and the holographic principle only a remarkable coincidence or is it the manifestation of a profound physical property of gravity time-dependent models, CFT's and the holographic principle? To answer this question it is then natural to try to generalize these fundamental conceptions to more general physical configurations. In this context, Wang et al. [9] have generalized the Cardy-Verlinde formula to the case of universes with a cosmological constant, Nojiri et al. [10] have found quantum bounds for that formula, Youm 11] has generalized the entropy formula to include perfect fluids with a general barotropic equation of state, Brevik and Odintsov [12, 13] considered generalizations with a constant bulk viscosity. A more extensive list of recent works can be found in 11].

The question we would like to answer is if the previous results can be generalized to any dimension and various time-dependent metric components. In this paper, we restrict ourselves to the analysis, in Section II, of the Bianchi type IX cosmological model with a perfect fluid as source. As is well known, this model reduces to the closed FRW model in the isotropic limit. In Section III, we use the first law of thermodynamics together with the energy conservation law in order to obtain an expression for the entropy which turns out to be a generalization of the Cardy-Verlinde entropy formula. Then, in Section IV, we assume the validity of the Cardy entropy formula and show that it represents the Hamiltonian constraint of the field equations, when the Virasoro operator and the central charge are defined in a suitable way. This allows us to propose definitions of the Bekenstein, Bekenstein-Hawking and Hubble entropies, which turn out to depend explicitly on the cosmological anisotropies. We compare the results given in these last two sections and discuss the validity of the Cardy-Verlinde formula for cosmological models with different expansion factors. Section V 
is devoted to conclusions.

\section{THE BIANCHI TYPE IX MODEL}

As a particular case of a gravity time-dependent model, let us consider the general line element for the Bianchi type IX cosmological model [14]

$$
d s^{2}=d t^{2}-e^{-2 \Omega}\left[e^{2 X+2 Y}\left(\omega^{1}\right)^{2}+e^{2 X-2 Y}\left(\omega^{2}\right)^{2}+e^{-4 X}\left(\omega^{3}\right)^{2}\right]
$$

where $\Omega, X$, and $Y$ are functions of the time $t$ only and

$$
\begin{gathered}
\omega^{1}=\frac{1}{2}\left(-\sin x^{3} d x^{1}+\sin x^{1} \cos x^{3} d x^{2}\right) \\
\omega^{2}=\frac{1}{2}\left(\cos x^{3} d x^{1}+\sin x^{1} \sin x^{3} d x^{2}\right) \\
\omega^{3}=\frac{1}{2}\left(\cos x^{1} d x^{2}+d x^{3}\right)
\end{gathered}
$$

where $\left(x^{1}, x^{2}, x^{3}\right)$ are the Euler angles of $S O(3)$. In order to write the corresponding field equations in a form more suitable for our analysis, we introduce the scale factors

$$
a_{1}=e^{-\Omega+X+Y}, \quad a_{2}=e^{-\Omega+X-Y}, \quad a_{3}=e^{-\Omega-2 X}
$$

with their corresponding Hubble parameters $H_{i}=\dot{a}_{i} / a_{i}(i=1,2,3)$. Using then the relationships $H_{1}+H_{2}+H_{3}=-3 \dot{\Omega}, H_{1}+H_{2}-2 H_{3}=6 \dot{X}$, and $H_{1}-H_{2}=2 \dot{Y}$, we can write the Hamiltonian constraint as

$$
\frac{1}{3}\left(H_{1} H_{2}+H_{1} H_{3}+H_{2} H_{3}\right)+\frac{1}{a_{1}^{2}}\left(1+\frac{1}{3} \epsilon^{2}\right)=\frac{8}{3} \pi G \rho
$$

with

$$
\epsilon^{2}=1-\frac{a_{3}^{2}}{a_{2}^{2}}-2\left(1-\frac{a_{1}^{2}}{a_{2}^{2}}\right)-\frac{a_{2}^{2}}{a_{3}^{2}}\left(1-\frac{a_{1}^{2}}{a_{2}^{2}}\right)^{2} .
$$

Furthermore, the remaining dynamical equations can be put in the form

$$
\begin{aligned}
& \dot{H}_{1}+\dot{H}_{3}+H_{1}^{2}+H_{3}^{2}+H_{1} H_{3}+\frac{a_{1}^{2}}{a_{2}^{2} a_{3}^{2}}-3 \frac{a_{2}^{2}}{a_{1}^{2} a_{3}^{2}}+\frac{a_{3}^{2}}{a_{1}^{2} a_{2}^{2}}+2 \frac{1}{a_{1}^{2}}-2 \frac{1}{a_{2}^{2}}+2 \frac{1}{a_{3}^{2}}=-8 \pi G p, \\
& \dot{H}_{2}+\dot{H}_{3}+H_{2}^{2}+H_{3}^{2}+H_{2} H_{3}-3 \frac{a_{1}^{2}}{a_{2}^{2} a_{3}^{2}}+\frac{a_{2}^{2}}{a_{1}^{2} a_{3}^{2}}+\frac{a_{3}^{2}}{a_{1}^{2} a_{2}^{2}}-2 \frac{1}{a_{1}^{2}}+2 \frac{1}{a_{2}^{2}}+2 \frac{1}{a_{3}^{2}}=-8 \pi G p, \\
& \dot{H}_{1}+\dot{H}_{2}+H_{1}^{2}+H_{2}^{2}+H_{1} H_{2}+\frac{a_{1}^{2}}{a_{2}^{2} a_{3}^{2}}+\frac{a_{2}^{2}}{a_{1}^{2} a_{3}^{2}}-3 \frac{a_{3}^{2}}{a_{1}^{2} a_{2}^{2}}+2 \frac{1}{a_{1}^{2}}+2 \frac{1}{a_{2}^{2}}-2 \frac{1}{a_{3}^{2}}=-8 \pi G p
\end{aligned}
$$


where $\rho$ is the matter density, $p$ its pressure, and $G$ denotes Newton's gravitational constant. An overdot represents differentiation with respect to the time $t$.

From the dynamical field equations (86)-(10) we obtain the energy-momentum conservation law which in this case is given by

$$
\dot{\rho}-3 \dot{\Omega}(\rho+p)=0
$$

For a barotropic equation of state $p=\omega \rho$ the last equation can be integrated and yields

$$
\rho=\rho_{0} e^{3(1+\omega) \Omega}
$$

where $\rho_{0}$ is a constant.

\section{THE ENTROPY FROM THE FIRST LAW OF THERMODYNAMICS}

Let us now suppose that the Bianchi type IX model satisfies the first law of thermodynamics, $T d S=d E+p d V$, where $T$ is the temperature of the universe and $S$ is the total entropy contained inside the volume $V$. For a comoving volume element of unit coordinate volume and physical volume $v$, from the first law of thermodynamics we obtain

$$
\dot{s}=\frac{v}{T}\left[\dot{\rho}+(p+\rho) \frac{\dot{v}}{v}\right]
$$

where $s$ is the entropy density per comoving volume. A reasonable way for defining the physical volume is as $v=a_{1} a_{2} a_{3}=e^{-3 \Omega}$, an expression which coincides with the physical volume of the Friedman-Robertson-Walker universe due to the special form of the anisotropies in the Bianchi type IX model. This assumption is supported also by the fact that the total volume of the universe turns out to be $V=\int \sqrt{-h} d^{3} x=\frac{1}{8} e^{-3 \Omega} \int \sin x_{1} d^{3} x$, where $h$ is the determinant of the spatial part of the line element (11). Therefore, we can write $V=\kappa v=\kappa e^{-3 \Omega}$, where $\kappa$ is a constant resulting from the previous integral. From Eq.(13) and the energy-momentum conservation law (11) it follows that the entropy density remains invariant $(\dot{s}=0)$ during the evolution, i.e., the expansion is adiabatic. Furthermore, the

first law of thermodynamics can be integrated for $s$ and yields [1]

$$
s=\frac{e^{-3 \Omega}}{T}(p+\rho)+s_{0}
$$

where $s_{0}$ is an additive constant. 
Following Verlinde's approach [2] we represent the total energy $E=\rho V$ as the sum of an extensive energy $E_{E}(S, V)$ and a sub-extensive energy $E_{C}(S, V)$

$$
E=E_{E}+\frac{1}{2} E_{C}
$$

where $E_{C}$ is the Casimir energy which is defined as the violation of the Euler identity:

$$
E_{C}=3(E+p V-T S)
$$

Under a scale transformation $S \rightarrow \lambda S$ and $V \rightarrow \lambda V$ the components of the energy behave as $E_{E}(\lambda S, \lambda V)=\lambda E_{E}(S, V)$ and $E_{C}(\lambda S, \lambda V)=\lambda^{1 / 3} E_{C}(S, V)$.

Introducing the expression for the entropy (14) into Eq.(16) we obtain

$$
E_{C}=-3 \frac{s_{0}}{s-s_{0}}(p+\rho) V
$$

or

$$
E_{C}=-3 \kappa \frac{s_{0} \rho_{0}}{s-s_{0}}(1+\omega) e^{3 \omega \Omega}
$$

for a universe satisfying a barotropic equation of state. Therefore, the behavior of the Casimir energy during the evolution in time is given by $E_{C} \sim e^{3 \omega \Omega}$. On the other hand, we see from Eq.(12) that the total energy of the system is given by $E=\kappa \rho_{0} e^{3 \omega \Omega}$ and, consequently, the extensive energy behaves similarly, $E_{E} \sim e^{3 \omega \Omega}$.

The total entropy $S$ of the universe should be related to the energy in such a way that the adiabaticity condition derived above holds. Therefore, if we associate the entropies $S_{E}$ and $S_{C}$ to the extensive and Casimir energy, respectively, from the energy behavior given above we conclude that only the expressions $S_{E} \sim E_{E} e^{-3 \omega \Omega}$ and $S_{C} \sim E_{C} e^{-3 \omega \Omega}$ satisfy this condition. Furthermore, taking into account the behavior of the energies under a scale transformation one can derive their dependence in terms of the entropy. These expressions can be written in a convenient form as

$$
E_{E}=\frac{\alpha}{4 \pi} e^{3 \omega \Omega} S^{1+\omega}, \quad E_{C}=\frac{\beta}{2 \pi} e^{3 \omega \Omega} S^{1 / 3+\omega}
$$

where $\alpha$ and $\beta$ are arbitrary constants (In CFT's one has that $\sqrt{\alpha \beta}=3$ as a consequence of the AdS/CFT correspondence). Then, the total entropy is related to the energy as [1]

$$
S=\left[\frac{2 \pi}{\sqrt{\alpha \beta}} e^{-3 \omega \Omega} \sqrt{2 E_{E} E_{C}}\right]^{\frac{3}{2+3 \omega}} .
$$


This expression for $S$ represents the generalization of the original Cardy-Verlinde formula to the case of an anisotropic Bianchi IX model, and reduces to it only in the case of a radiative universe $(\omega=1 / 3)$ in accordance to the results in [2].

Equation (20) defines the total entropy of a universe described by a Bianchi type IX model. It resembles the entropy for a FRW universe. One could wonder where the timedependent scale factors of the Bianchi model are present in this expression. In fact, they are not. The reason is that in the analysis based upon the first law of thermodynamics, the explicit form of the total volume $V$ and the physical volume $v$ is crucial and, as we have shown, they do not depend explicitly on the functions $X$ and $Y$ which determine the three different rates of expansion.

Notice that for a fixed value of $E$ and positive $\omega$ the entropy (20) has a maximum value so that the bound

$$
S \leq\left[\frac{2 \pi}{\sqrt{\alpha \beta}} E e^{-3 \omega \Omega}\right]^{\frac{3}{2+3 \omega}}
$$

holds. This becomes the Bekenstein bound, $S \leq S_{B}$ with $S_{B}=\frac{2 \pi}{3} E e^{-\Omega}$ for a radiation dominated universe with $\omega=1 / 3$ and normalization factor $\sqrt{\alpha \beta}=3$. It seems therefore appropriate to introduce

$$
S_{B}^{f l t}=\left[\frac{2 \pi}{3} E e^{-3 \omega \Omega}\right]^{\frac{3}{2+3 \omega}}
$$

as the Bekenstein entropy which bounds the total entropy of the Bianchi type IX cosmological model. The superscript " $f l t$ " means that this expression for the entropy has been obtained by using the first law of thermodynamics, and will be helpful when comparing it with a different expression which will be derived in the next section. If we insert into Eq.(22) the

expression for the total energy, we find that $S_{B}^{f l t}$ remains constant during the cosmological evolution. Hence, the Bekenstein entropy represents a constant bound for the total entropy of the system.

\section{THE ENTROPY FROM CFT}

The universal validity of the Cardy entropy formula

$$
S_{C}=2 \pi \sqrt{\frac{c}{6}\left(L_{0}-\frac{c}{24}\right)},
$$

for CFT's has been proposed in [2] by using a definition of the central charge $c$ in terms of the Casimir energy. We intentionally use here the notation $S_{C}$ for the Cardy entropy in 
order to distinguish it from the entropy $S$ derived in the last section by means of the first law of thermodynamics. Let us assume here that this entropy formula is valid for the Bianchi type IX model. Then, independently of the equation of state governing the evolution of this cosmological model, it can easily be seen that the Hamiltonian constraint (6) coincides with Eq.(23) if the eigenvalue $L_{0}$ of the Virasoro operator and the central charge $c$ are chosen as

$$
L_{0}=\frac{1}{3} E \frac{a_{1}}{\sqrt{1+\epsilon^{2} / 3}}, \quad c=\frac{3}{\pi G} \frac{V}{a_{1}} \sqrt{1+\epsilon^{2} / 3},
$$

Furthermore, the Cardy entropy is related to the Hubble parameters as

$$
S_{C}=\frac{1}{2 \sqrt{3} G} \sqrt{H_{1} H_{2}+H_{1} H_{3}+H_{2} H_{3}} V .
$$

Notice that the explicit values given in Eqs.(24)-(25) can formally be obtained by applying the transformation

$$
H \rightarrow \frac{1}{\sqrt{3}} \sqrt{H_{1} H_{2}+H_{1} H_{3}+H_{2} H_{3}}, \quad a \rightarrow \frac{a_{1}}{\sqrt{1+\epsilon^{2} / 3}},
$$

on the Hubble parameter $H$ and the scale factor $a$ of the isotropic FRW spacetime. Written in this form, this transformation seems to indicate the presence of a preferred direction determined by an inhomogeneity in $a_{1}$. However, similar expressions can be written for $a_{2}$ and $a_{3}$ (with the corresponding changes in the definition of $\epsilon^{2}$ ) and, therefore any of the directions could have been chosen. The specific direction chosen in order to perform the transformation (26) is only a matter of conventions. Also, it should be mentioned that once we assume that the Cardy formula (23) is valid for the Bianchi type IX model, and we demand that it represents the field equation (6), then the identification of $L_{0}$ and $c$ given in (24) is unique (modulo the convention mentioned above).

In a similar manner, the Hamiltonian constraint (6) can be rewritten as the Verlinde formula

$$
S_{H}^{2}-2 S_{B H} S_{B}+S_{B H}^{2}=0,
$$

where the Bekenstein, $S_{B}$, the Hubble, $S_{H}$, and the Bekenstein-Hawking entropy, $S_{B H}$, are given by

$$
\begin{aligned}
S_{B} & =\frac{2 \pi}{3} \frac{E a_{1}}{\sqrt{1+\epsilon^{2} / 3}}, \\
S_{B H} & =\frac{1}{2 G} \frac{V}{a_{1}} \sqrt{1+\epsilon^{2} / 3},
\end{aligned}
$$




$$
S_{H}=\frac{1}{2 \sqrt{3} G} \sqrt{H_{1} H_{2}+H_{1} H_{3}+H_{2} H_{3}} V .
$$

Then, by analogy with the cases studied in other works (see 11] for a list of references) in which the relationship between the Cardy formula and the field equations has been investigated, we propose expressions (28)-(30) as the definitions of the entropies for the Bianchi type IX model. In the isotropic limit $\left(\epsilon^{2}=0\right)$ we recover the corresponding entropies for the FRW model.

Eq.(27) has exactly the same Pythagorean form as that found for the FRW model [2]. This allows us to represent the dynamical evolution of the entropies by a circle of radius $S_{B}$ which, in contrast to the FRW case, is not constant but changes as time evolves. As a consequence, the dynamical evolution of any of the entropies defined in Eqs.(28)-(30), which is governed by the field equations (18)-(10), is no longer determined by the remaining entropies but depends on the specific characteristics of the fluid. For instance, from Eqs.(28) and (29) and using the field equations (81)-(10) in the form (11) one can show that

$$
\frac{d}{d t}\left(S_{B} S_{B H}\right)=3(\omega-1) \dot{\Omega} S_{B} S_{B H}
$$

On the other hand, from Eq.(27) we obtain

$$
\dot{S}_{H}=\frac{S_{B H}}{S_{H}}\left[3(\omega-1) \dot{\Omega} S_{B}-\dot{S}_{B H}\right] .
$$

The last two equations determine the dynamical behavior of the entropies which will then depend on the specific value of $\omega$. The case $\omega=1$ can easily be solved, however it is not of particular interest in this work.

To get some insight into the structure of these entropies, one can calculate the approximate contribution for the special case in which one has only two different time-dependent metric components. This can be reached by considering $X$ as an infinitesimal quantity $(X<<1)$ and assuming $Y=0$. Then we have

$$
\begin{aligned}
S_{B}^{a} & =S_{B}^{i}\left(1+\frac{5}{2} X^{2}\right), \\
S_{B H}^{a} & =S_{B H}^{i}\left(1-\frac{5}{2} X^{2}\right), \\
S_{H}^{a} & =S_{H}^{i}\left(1-\frac{\dot{X}^{2}}{2 H^{2}}\right),
\end{aligned}
$$

where the superscripts $a$ and $i$ denote the corresponding "anisotropic" and "isotropic" quantities, respectively. 
The first thing one can notice is that, due to the anisotropies, the Bekenstein entropy given in Eq.(28) does not remain constant during the evolution in time [cf. also Eq.(33)]. This differs from the result obtained in the last section for the Bekenstein entropy $S_{B}^{f l t}$ which is a constant that only depends on the type of fluid (i.e., it depends on $\omega$ ). Nevertheless, both entropies (22) and (28) reduce in the limiting case $X=0, Y=0, \omega=1 / 3$ to the Bekenstein entropy of a radiation dominated FRW universe.

In terms of the Hubble entropy, $S_{H}$, and the Bekenstein-Hawking energy, $E_{B H}$, which are defined as

$$
S_{H}=S_{C}, \quad E_{B H}=\frac{3}{4 \pi G} \frac{V}{a_{1}^{2}}\left(1+\epsilon^{2} / 3\right),
$$

Eq.(23) for the Bianchi type IX model can be identified with the cosmological Cardy formula

$$
S_{H}=\frac{2 \pi}{3} \frac{a_{1}}{\sqrt{1+\epsilon^{2} / 3}} \sqrt{E_{B H}\left(2 E-E_{B H}\right)} .
$$

In a radiation dominated FRW universe the Cardy-Verlinde formula (201) coincides with the cosmological Cardy formula (37), with $E_{B H}$ playing the role of the Casimir energy $E_{C}$. This is not true in our general case. Whereas the functional dependence in the cosmological Cardy formula (37) is dictated by the square root of the energies, independently of the equation of state, this functional dependence in the Cardy-Verlinde formula (201) is different for each equation of state and becomes a square root only for $\omega=1 / 3$. Let us therefore consider only the case of a radiation dominated Bianchi IX universe, for which the Cardy-Verlinde formula (20) reads (we take $\sqrt{\alpha \beta}=3$ and use the relationship $E_{E}=\left(2 E-E_{C}\right) / 2$ )

$$
S=\frac{2 \pi}{3} e^{-\Omega} \sqrt{E_{C}\left(2 E-E_{C}\right)},
$$

and for this case let us investigate the cosmological bound on the Casimir energy

$$
E_{C} \leq E_{B H}
$$

postulated by Verlinde [2]. From Eq.(18) we see that $E_{C} \sim e^{\Omega}$ while the Bekenstein-Hawking entropy behaves like $E_{B H} \sim e^{-\Omega-2 X-2 Y}\left(1+\epsilon^{2} / 3\right)$, according to Eq.(36). Thus, the Verlinde energy bound (39) can be satisfied, but a difference appears in the saturation. Consider, for instance, the approximate case $(Y=0, X<<1)$. Using the approximate expression (34) we get $E_{B H}^{a}=(3 / 4 \pi G) e^{-\Omega}\left(1-8 X^{2}\right)=E_{B H}^{i}\left(1-8 X^{2}\right)<E_{B H}^{i}$. On the other hand, according to Eq.(18) $E_{C}^{a}=E_{C}^{i}$. Since the bound $E_{C}^{i} \leq E_{B H}^{i}$ holds and becomes saturated for 
a specific size of an expanding universe, then the bound $E_{C}^{a} \leq E_{B H}^{a}$ will be the generalization of the isotropic case. However, because $E_{B H}^{a}=E_{B H}^{i}\left(1-8 X^{2}\right)$, the previous relation, for the anisotropic case means that the bound would become saturated for a larger size, compared with the isotropic case, of the expanding universe. At the saturation point $E_{C}^{a}=E_{B H}^{a}$, however, the Cardy-Verlinde formula (38) does not coincide with the cosmological Cardy formula (37), because of the presence of the various scale factors entering the last formula. In fact, for the approximate case we obtain that $S_{H}=S\left(1+5 / 2 X^{2}\right)$ and the coincidence breaks down.

\section{CONCLUSIONS}

In this work we have pursued the definition of the entropies associated with gravity time-dependent models. These entropies have been found for the Bianchi type IX model. Since this model is considered the anisotropic generalization of the closed FRW model, it should give us the modified entropies due to the presence of several time-dependent metric components. First, we assumed that the universes described by this model satisfy the first law of thermodynamics. The resulting entropy is given in Eq.(20) and represents a generalization of the Cardy-Verlinde formula for these models. Remarkably, this entropy remains constant during the evolution of time as a consequence of the energy conservation law and the assumed first law of thermodynamics.

Secondly, we assume the universal validity of the Cardy entropy formula and show that it coincides with the Hamiltonian constraint of the field equations. This allows us to consider the cosmological Cardy formula (37) and define the Bekenstein, Bekenstein-Hawking and Hubble entropies. The first obvious consequence is that these entropies do depend on the different metric components.

The straightforward generalization of the Verlinde energy bound for the anisotropic radiative case $\left(E_{C}^{a} \leq E_{B H}^{a}\right)$ indicates that the saturation takes place for a universe larger than the one in the corresponding isotropic case. We also have shown that the Cardy-Verlinde formula (38) and the cosmological Cardy formula (37) do not coincide when the bound is saturated $\left(E_{C}^{a}=E_{B H}^{a}\right)$.

An elegant AdS/CFT prescription has been proposed [15] (see also [16] for a review) in which starting with a Schwarzschild $\mathrm{AdS}_{5}$ metric and specifying the location of a brane in 
parametric form one can induce a 4-dimensional FRW radiation universe metric on the brane. In [17] and 18] this approach has been generalized to include the cases of asymptotically flat and asymptotically AdS charged black holes. This has shed new light on Verlinde's proposal and on the coincidence between the CFT entropy formulae and the FRW equations at the horizon. The case of models with various scale factors has been less explored. Nevertheless, a geometric braneworld description of the Bianchi $\mathrm{VI}_{-1}$ model was obtained in [19] from other solutions found to the vacuum Einstein equations with negative cosmological constant in 5-dimensions. However, generalized entropies have not been investigated and it is not clear if they could be defined for this case. On the other hand, as mentioned above, the Bianchi IX model contains the closed FRW model in the isotropic limit and one can study the influence of several metric components on the entropies. Nonetheless, the search for a higher dimensional static metric by means of which one could get the Bianchi IX model on the brane is a matter of future work.

On the other hand, in this work we have analyzed only the Bianchi type IX model. It seems reasonable to expect that the results obtained here can be generalized to include other Bianchi type A models [20]. Moreover, the results presented here can, in principle, be generalized to any number of dimensions with several time-dependent metric components. However, the entropy expresions (20) and (22) reduce to the original Cardy-Verlinde formula only for a radiative universe. A next step would be to try to extend the kind of results obtained here for various gravity time-dependent components to their study in relation with more general matter fields [10, 11, 12, 13]. This would allow the study of other physical interesting systems like SD-branes [21], the kind of objects that are constructed by means of time-dependent metric components and that arise naturally in string theory.

\section{Acknowledgments}

We would like to thank M. P. Ryan for helpful discussions. This work was supported by DGAPA-UNAM grant IN112401, CONACyT-Mexico grants 36581-E, 37851-E, and sabbatical grant 020331 (O. O.). L. P. was supported by a UNAM-DGEP Graduate Fellowship.

[1] E. Kolb and M. Turner, The Early Universe (Addison-Wesley, 1990). 
[2] E. Verlinde, On the holographic principle in a radiation dominated universe, hep-th/0008140.

[3] J. D. Bekenstein, Lett. Nuovo Cim. 4737 (1972).

[4] S. Hawking, Commun. Math. Phys. 43199 (1975).

[5] G. t'Hooft, in: Salamfestschrift: A collection of talks, edited by A. Ali, J. Ellis, and S. Randjbar-Daemi (World Scientific, Singapore, 1993), gr-qc/9310026

[6] L. Susskind, J. Math. Phys. 366377 (1995).

[7] W. Fischler and L. Susskind, Holography and cosmology, hep-th/9806039.

[8] J. L. Cardy, Nucl. Phys. B 270967 (1986).

[9] E. Wang, E. Abdalla and R. Su, Phys. Lett. B 503394 (2001).

[10] O. Nojiri, S. Odintsov, O. Obregon, H. Quevedo and M. Ryan, Mod. Phys. Lett. A 161181 (2001).

[11] D. Youm, Phys. Lett. B 531, 276 (2002).

[12] I. Brevik and S. D. Odintsov, Phys. Rev. D 65067302 (2002).

[13] I. Brevik, Phys. Rev. D 65127302 (2002).

[14] C. W. Misner, K. S. Thorne and J. A. Wheeler, Gravitation (W. H. Freeman and Company, San Francisco, 1973).

[15] I. Savonije and E. Verlinde, Phys. Lett. B 507305 (2001).

[16] F. Quevedo, Class. Quant. Grav. 195721 (2002).

[17] D. Youm, Mod. Phys. Lett. A 161263 (2001).

[18] D. Youm, Mod. Phys. Lett. A 161327 (2001).

[19] C. Cadeau and E. Woolgar. Class. Quant. Grav. 18527 (2001).

[20] M. P. Ryan and L. C. Shepley, Homogeneous relativistic cosmologies (Princeton University Press, Princeton, 1975).

[21] M. Gutperle and A. Strominger, J. High Energy Phys. JHEP 0204018 (2002); see also A. Sen, J. High Energy Phys. JHEP 0204048 (2002), F. Leblond and A. W. Peet, SD-brane gravity fields and rolling tachyons, hep-th/0303035, and references therein. 